\title{
The effects of various estrogen doses on the proliferation and differentiation of cultured neural stem cells
}

\author{
Lixia Zhang ${ }^{1, *}$, Yaqun $\mathrm{Ma}^{2, *}$, Min Liü ${ }^{3, *}$, Yulong $\mathrm{Ma}^{3}$ and Hang $\mathrm{Guo}^{2}$ \\ ${ }^{1}$ Department of Burn and Plastic Surgery, The Fourth Medical Center to Chinese People's Liberation Army (PLA) General \\ Hospital, Beijing, China \\ ${ }^{2}$ Department of Anesthesiology, The Seventh Medical Center to Chinese PLA General Hospital, Beijing, China \\ ${ }^{3}$ Anesthesia and Operation Center, The First Medical Center to Chinese PLA General Hospital, Beijing, China
}

\begin{abstract}
The brain has long been known as a dimorphic organ and as a target of estrogen. Neurogenesis, including proliferation and differentiation of neural stem cells (NSCs), could be stimulated and regulated by estrogen. However, the dose and timing of estrogen treatment is controversial, and the underlying mechanism remains unclear. In this study, we tested the effects of various estrogen doses on the neurogenesis of NSCs derived from Sprague-Dawley rat embryos. First, we identified that the estrogen receptor-ER $\alpha, E R \beta$ and GPR30 were highly expressed in NSCs. The results from cell cycle and Western blot analyses revealed that $10 \mathrm{nM} 17 \beta$-estradiol (E2) treatment for 3 days significantly increased NSCs proliferation of and p-ERK1/2 expression level but that $50 \mathrm{nM}$ E2 exposure markedly decreased NSCs proliferation and p-ERK1/2 expression level. According to immunofluorescence staining and Western blot analyses, $10 \mathrm{nM} \mathrm{E} 2$ treatment for 7 days significantly stimulated NSCs to differentiate into neurons and inhibited their differentiation into astrocytes. These results demonstrate that NSCs are a target of estrogen and that an appropriate dose of E2 (10 nM) can significantly increase the proliferation of NSCs and stimulate NSCs to differentiate into neurons, which contributes to knowledge regarding the regulatory effects of estrogens on neurogenesis.
\end{abstract}

Key words: Estrogen - Neurogenesis - Neural stem cells - Proliferation — Differentiation

Abbreviations: BrdU, bromodeoxyuridine; CNPase, 20,30-cyclic nucleotide 30-phosphodiesterase; DAPI, diamidino phenylindole; E2, 17 $\beta$-estradiol; ER, estrogen receptor; GFAP, glial fibrillary acid protein; MSNs, medium spiny neurons; NSCs, neural stem cells; PLA, People's Liberation Army; ERK, extracellular signal-regulated kinase; Tuj1, neuronal class III $\beta$-tubulin.

\section{Introduction}

For many years, estrogens were thought of only as "sex hormones" that function in the reproductive system of

\footnotetext{
* These authors contributed equally to this study.

Correspondence to: Hang Guo, Department of Anesthesiology, The Seventh Medical Center to Chinese PLA General Hospital, Beijing, China

E-mail: gh_wyb@126.com

Yulong Ma, Anesthesia and Operation Center, The First Medical Center to Chinese PLA General Hospital, Beijing, China

E-mail: yulongma123@163.com
}

animals. However, there is ample empirical evidence to support the notion that the biological impact of estrogens extends beyond the gonads to other organs, including the brain, and behavior (Rettberg et al. 2014). Sex-specific shifts in endogenous estrogen levels related to menstrual cycle, pregnancy, and menopause are also associated with differences in multiple brain functions (Workman et al. 2012).

Neurogenesis in the adult brain, which can be stimulated by physiological factors, such as growth factors and environmental cues, and by pathological processes, including stroke and neurodegeneration (Shao et al. 2012), is considered to be an important pathway for neuroprotection and neurological recovery. Neurogenesis continues throughout life in specific regions of the mammalian brain, including the dentate gyrus 
(DG) of the hippocampus. These newborn cells can migrate into damaged brain regions and differentiate into neural cells to alleviate neural injury (Jin et al. 2003). Therefore, neural stem cells (NSCs) have become the target for neuroprotection. NSCs have unique properties, including pluripotency, and hold promise for neurodevelopmental biology, regenerative medicine and drug discovery.

Nevertheless, the factors governing the fates of NSCs are still poorly understood. Estrogens exhibit neuroprotective effects by promoting neurogenesis (Bourque et al. 2009; Pike et al. 2009; Li et al. 2011). Thus, estrogens may play a profound role in the modulation of NSCs, and NSC transplantation combined with estrogen modulation may be a therapeutic approach for neurodegenerative diseases. However, the underlying mechanisms remain unclear.

The most important characteristics of NSCs are proliferation and controlled differentiation. Therefore, in this study, we investigated the effects of different estrogen doses on the proliferation and differentiation of NSCs.

\section{Materials and Methods}

The experimental protocol was approved by the Ethics Committee for Animal Experimentation of Chinese People's Liberation Army (PLA) General Hospital (Beijing, China) and performed in accordance with the guidelines for Animal Experimentation of Chinese PLA General Hospital.

\section{Isolation and culture of NSCs}

NSCs were harvested from the brains of E14.5-E16.5 SpragueDawley rat embryos (from pregnant dames purchased from the Experimental Animal Center of the Chinese PLA General Hospital) as previously described (Nie et al. 2013). Briefly, hippocampi were isolated in ice-cold dissection buffer (HBSS, Gibco, USA) under a stereomicroscope. After the meninges were removed, single-cell suspensions were obtained by mechanical dissociation. Cells were washed, briefly centrifuged, resuspended in fresh medium, and cultured at $5 \times 10^{5}$ cells $/ \mathrm{ml}$ in $25-\mathrm{cm}^{2}$ cell culture flasks (Corning, USA) in serum-free Dulbecco's modified Eagle's medium (DMEM)/F12 medium (1:1 mixture of DMEM and Ham's F12, Gibco, USA) supplemented with $20 \mathrm{ng} / \mathrm{ml}$ basic fibroblast growth factor (bFGF, Peprotech, USA), $20 \mathrm{ng} / \mathrm{ml}$ epidermal growth factor (EGF, Peprotech, USA), 2\% B-27 and 1\% N-2 supplements (Gibco, USA), $1 \%$ penicillin and $1 \%$ streptomycin. The resulting neurospheres were harvested and mechanically dissociated to produce single-cell suspensions for replating every 6-7 days.

To verify the identity of NSCs, we dissociated neurospheres and plated them onto poly-L-lysine-coated glass coverslips. After a $24 \mathrm{~h}$ attachment period, the cells were fixed in $4 \%$ paraformaldehyde for $45 \mathrm{~min}$ and processed for nestin im- munostaining. The slides and coverslips were rinsed in phosphate-buffered saline (PBS) and then incubated with a mouse anti-nestin antibody (1:500, Abcam, USA) overnight at $4^{\circ} \mathrm{C}$. Immunolabeling was visualized with anti-mouse Alexa Fluor 594-conjugated secondary antibody (1:500, Invitrogen, USA).

To estimate the rate of proliferation, we plated neurospheres in serum-free medium with bFGF. To induce differentiation, we changed the medium to DMEM/F12 containing $4 \%$ fetal bovine serum (Sigma-Aldrich, USA) without bFGF.

\section{Estrogen exposure}

Neurospheres were exposed to $17 \beta$-estradiol (E2, Cayman, USA) at different physiological concentrations $(0,1,10,20$ and $50 \mathrm{nM}$ ) and durations (3 days for cell cycle analyses and 7 days for differentiation analyses). Dimethyl sulfoxide (DMSO) was used as the vehicle control; E2 was dissolved in DMSO in $10 \mathrm{mM}$ stock solutions. Further dilutions were prepared using culture medium. The final concentration of DMSO in the culture medium never exceeded $0.02 \%$, a level that has no effect by itself. Control group did not contain DMSO.

\section{Cell cycle analysis}

Cell cycle distribution was assessed by flow cytometry as previously described (Majewski et al. 2011). After 3 days of E2 exposure, cells were collected by trypsinization and centrifuged twice in PBS. The cells were then fixed in precooled $70 \%$ ethanol at $-20^{\circ} \mathrm{C}$ and stained with propidium iodide (PI) solution. DNA content was determined by flow cytometry using CellQuest Software. A total of 10,000 events were counted for each sample (FACSCalibur, Becton-Dickinson, USA). The percentage of cells in a particular cell cycle phase was calculated with ModFit software (Becton-Dickinson). The growth rate was calculated as the percentage of cells in S+G2 phase.

\section{Immunofluorescence staining and cell differentiation assays}

Hippocampal NSCs were phenotyped 7 days after E2 exposure according to their protein marker expression. Cells were seeded onto poly-D-lysine-coated coverslips at $3 \times 10^{4}$ cells/well in 24-well plates in DMEM/F12 medium containing $4 \%$ fetal bovine serum. The cells were exposed to E2 as described. Twenty-four hours after replating or seven days after E2 exposure, the cells were fixed in $4 \%$ paraformaldehyde for $45 \mathrm{~min}$, washed 3 times with PBS, and incubated for $30 \mathrm{~min}$ at room temperature in a blocking solution composed of PBS plus $1 \%$ bovine serum albumin (BSA) and $0.3 \%$ Triton X-100. Cells were then incubated at $4^{\circ} \mathrm{C}$ overnight in rabbit anti-ERa (1:100, Abcam, UK), rabbit anti-ER $\beta$ (1:100, Abcam, UK), rabbit anti-GPR30 (1:100, Abcam, UK), mouse anti-neuronal class III $\beta$-tubulin (Tuj1, 
1:500, Sigma-Aldrich, USA), rabbit anti-glial fibrillary acid protein (GFAP, 1:500, Millipore, USA), or mouse anti-20,30cyclic nucleotide 30-phosphodiesterase (CNPase, 1:200, Abcam, UK) antibodies diluted in the blocking solution. The cells were then incubated with secondary antibodies (1:500 Alexa Fluor 488 or Alexa Fluor 594, Invitrogen, USA) for $2 \mathrm{~h}$ at room temperature. Finally, the cells were incubated with diamidino phenylindole (DAPI, $1 \mu \mathrm{g} / \mathrm{ml}$ ) for $10 \mathrm{~min}$ at room temperature to stain the nuclei.

All stained cultures were analyzed under an FV-1000 laser scanning confocal microscope (Olympus, USA). Cell counting was performed using Image-Pro Plus version 6.0 software (Media Cybernetics, USA) by a researcher blinded to the experimental conditions. Cells were counted in 6-10 randomly chosen visual fields under a $10 \times$ or $20 \times$ objective. The percentage of positive cells is expressed as a fraction of the total number of DAPI-stained cells. At least 3 independent experiments were performed for each assay.

\section{Western blotting}

Cell samples were harvested from culture plates at $24 \mathrm{~h}$ after replating or 3 and 7 days after E2 exposure. The samples were lysed in sample buffer composed of $62.5 \mathrm{mM}$ Tris- $\mathrm{HCl}$, $2 \% \mathrm{w} / \mathrm{v}$ SDS, $10 \%$ glycerol, $50 \mathrm{mM}$ dithiothreitol (DTT), and $0.1 \% \mathrm{w} / \mathrm{v}$ bromophenol blue. Insoluble materials were separated by centrifugation at $12,000 \times \mathrm{g}$ for $10 \mathrm{~min}$, and the supernatant was heated to $100^{\circ} \mathrm{C}$ for $10 \mathrm{~min}$ and then cooled on ice for $30 \mathrm{~min}$. Electrophoresis was conducted by SDS-PAGE using 10\% polyacrylamide gels in accordance with routine protocols. Separated proteins were transferred onto nitrocellulose membranes, and the membranes were blocked (with gentle shaking) for $1 \mathrm{~h}$ at room temperature in a solution containing $5 \%$ nonfat milk powder and $0.1 \%$ Tween-20 in Tris-buffered saline (TBST). After washing 3 times in TBST, the membranes were incubated overnight at $4^{\circ} \mathrm{C}$ in blocking solution plus rabbit anti-ERa (1:500, Abcam, UK), rabbit anti-ER $\beta$ (1:500, Abcam, UK), rabbit anti-GPR30 (1:500, Abcam, UK), rabbit anti-phosphorylated extracellular signal-regulated kinases (p-ERK1/2, 1:2500, Cell Signaling, USA), rabbit anti-ERK1/2 (1:2500, Cell Signaling, USA), rabbit anti-Tuj1 (1:1000, Sigma-Aldrich, USA), or rabbit anti-GFAP (1:1000, Millipore, USA) antibodies. Mouse anti- $\beta$-actin (1:1000, Sigma-Aldrich, USA) and rabbit anti-GAPDH antibodies (1:1000, Sigma-Aldrich, USA) were used to identify $\beta$-actin and GAPDH, respectively, as loading controls. All membranes were then washed 3 times in PBS and incubated with peroxidase-conjugated goat anti-rabbit IgG or anti-mouse IgG in TBST for $1 \mathrm{~h}$. Immunoreactive bands were detected using enhanced chemiluminescence (ECL, Amersham, UK) and quantified with Bio-Rad Quantity One software (Hercules, CA, USA).
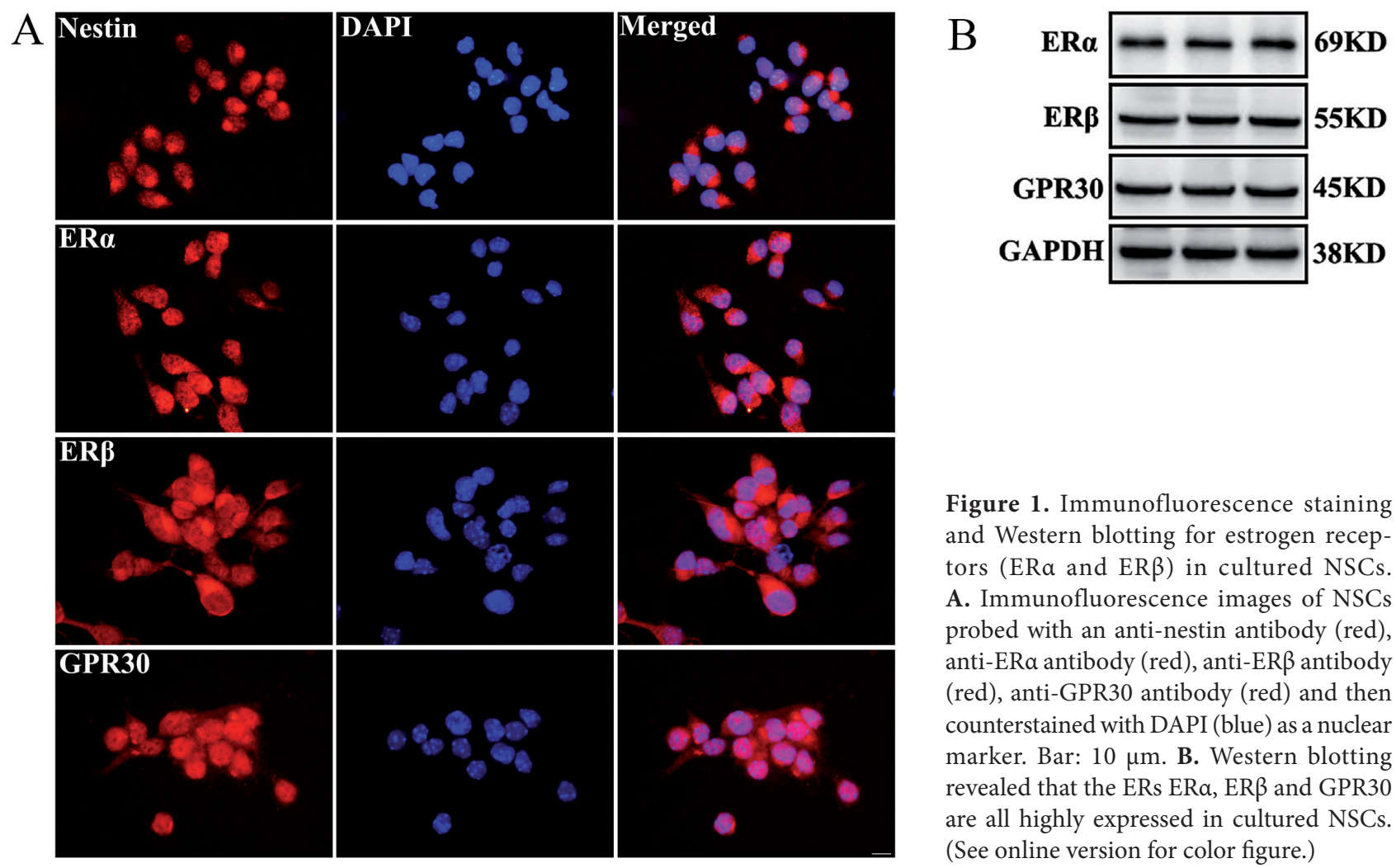

Figure 1. Immunofluorescence staining and Western blotting for estrogen receptors (ER $\alpha$ and ER $\beta$ ) in cultured NSCs. A. Immunofluorescence images of NSCs probed with an anti-nestin antibody (red), anti-ER $\alpha$ antibody (red), anti-ER $\beta$ antibody (red), anti-GPR30 antibody (red) and then counterstained with DAPI (blue) as a nuclear marker. Bar: $10 \mu \mathrm{m}$. B. Western blotting revealed that the ERs ER $\alpha$, ER $\beta$ and GPR30 are all highly expressed in cultured NSCs. (See online version for color figure.) 


\section{Statistical analysis}

The results are presented as means $\pm \mathrm{SD}$. Comparisons among multiple groups were performed using one-way ANOVA followed by Dunnett's post hoc test. GraphPad Prism 7.0 was used for statistical analyses. A $p$-value $<0.05$ was considered statistically significant. Data were collected by two independent investigators who were blinded to the drug treatments.

\section{Results}

\section{Identification of cultured NSCs}

Using our isolation and culture methods, most cells (>98\%) plated on poly-D-lysine-coated coverslips were immunopositive for the stem cell marker nestin $24 \mathrm{~h}$ after plating, confirming that the vast majority of cells used for subsequent assays were indeed NSCs (Figure 1A).
Estrogen receptors (ERs) are highly expressed in NSCs

As shown in Figure 1A, the results from immunofluorescence staining reveal that $\mathrm{ER} \alpha, \mathrm{ER} \beta$ and GPR30 are strongly expressed in the identified NSCs. Western blot analysis showed that ER $\alpha, E R \beta$ and GPR30 are highly expressed in the identified NSCs (Figure 1B).

\section{Effects of various E2 doses on NSC proliferation}

As shown in Figure 2, flow cytometric analysis indicated that the percentage of cells in S+G2 phase in the Control group and in NCs treated with 0,1 , and $20 \mathrm{nM} \mathrm{E2s} \mathrm{were} 26.4$ $\pm 2.7 \%, 27.7 \pm 2.0 \%, 28.1 \pm 2.5 \%$ and $29.1 \pm 2.6 \%$, respectively. No significant differences were observed among these groups. Treatment with $10 \mathrm{nM}$ E2 resulted in a significant increase in the percentage of cells in S+G2 phase (to 38.7 $\pm 3.5 \%,{ }^{* *} p=0.0012 v s$. the $0 \mathrm{nM}$ E2 group). Conversely, treatment with $50 \mathrm{nM}$ E2 induced a marked decrease in the
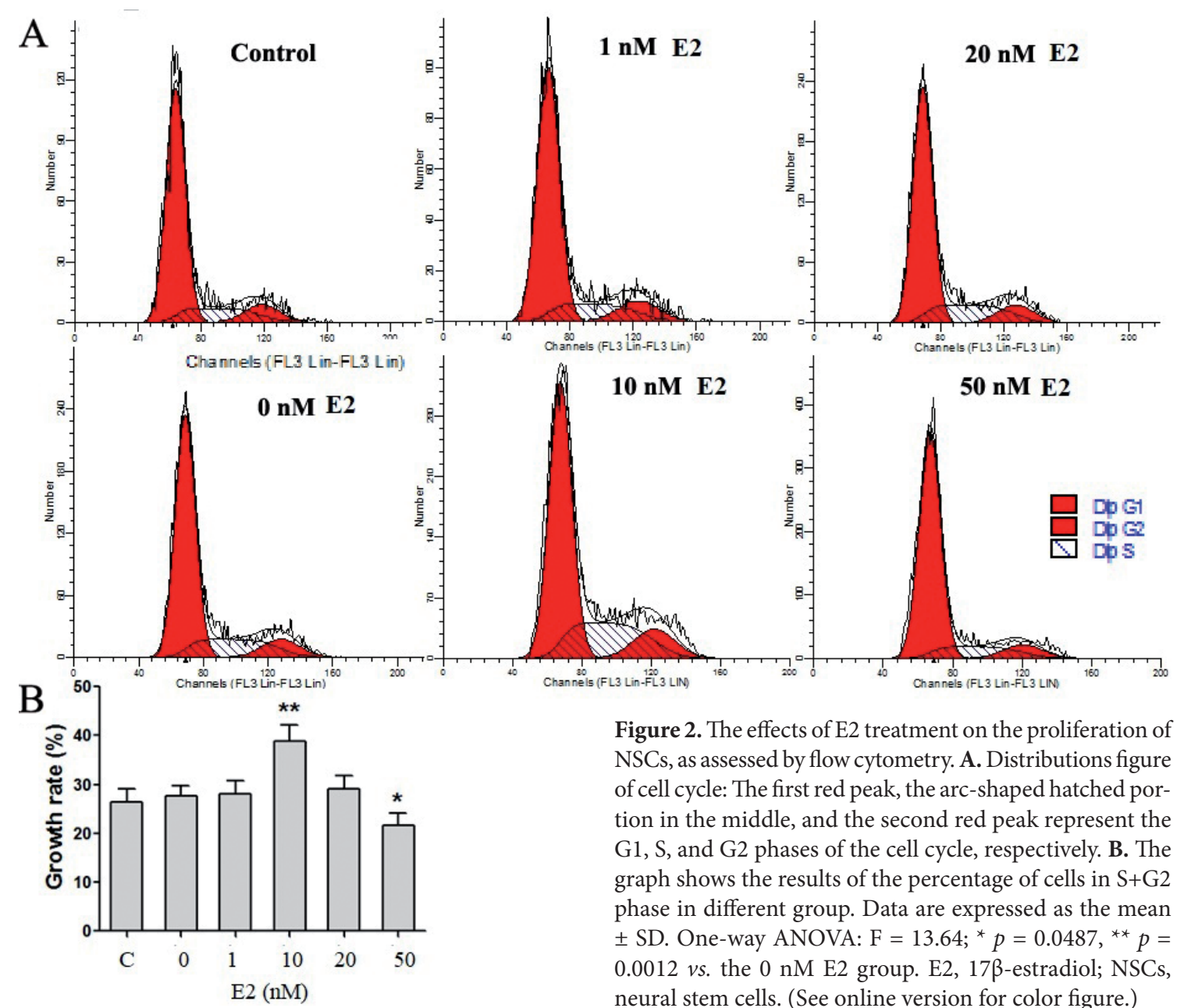

Figure 2. The effects of E2 treatment on the proliferation of NSCs, as assessed by flow cytometry. A. Distributions figure of cell cycle: The first red peak, the arc-shaped hatched portion in the middle, and the second red peak represent the G1, S, and G2 phases of the cell cycle, respectively. B. The graph shows the results of the percentage of cells in S+G2 phase in different group. Data are expressed as the mean \pm SD. One-way ANOVA: $\mathrm{F}=13.64 ;{ }^{*} p=0.0487,{ }^{*} p=$ $0.0012 v s$. the $0 \mathrm{nM} \mathrm{E2}$ group. E2, 17 $\beta$-estradiol; NSCs, neural stem cells. (See online version for color figure.) 
percentage of cells in $\mathrm{S}+\mathrm{G} 2$ phase $\left(21.4 \pm 2.6 \%,{ }^{*} p=0.0487\right.$ $v s$. the $0 \mathrm{nM}$ E2 group).

Effects of various E2 doses on p-ERK1/2 expression in NSCs

Next, we investigated the effects of various E2 doses on the ERK signaling cascade, which regulates proliferation. As shown in Figure 3 (Western blotting results), $10 \mathrm{nM}$ E2 treatment induced a significant increase in the level of p-ERK1/2 $\left({ }^{*} p=0.0174 v s\right.$. the $0 \mathrm{nM} \mathrm{E} 2$ group), whereas treatment with $50 \mathrm{nM}$ E2 markedly decreased the levels of p-ERK1/2 ( $p=$ $0.048 v$ s. the $0 \mathrm{nM}$ E2 group).

\section{Effects of various E2 doses on NSC differentiation}

To investigate whether $\mathrm{E} 2$ treatment induced the differentiation of cultured NSCs, we examined cell phenotypes 7 days after E2 treatment using the neuronal marker Tuj1, the astrocytic marker GFAP, and the oligodendrocytic marker CNPase. As shown in Figure 4, immunofluorescence assays revealed that compared to NCs treated with $0,1,20$ and $50 \mathrm{nM}$ E2 did not affect the percentage of Tuj1-positive or GFAP-positive cells in culture. However, $10 \mathrm{nM}$ E2 treatment significantly increased the percentage of Tuj1-positive cells (Figure $4 \mathrm{~B},{ }^{* * *} p=0.0001 v$ s. the $0 \mathrm{nM}$ E2 group) and decreased the percentage of GFAP-positive cells (Figure 4C, ${ }^{*} p=0.0484 v s$. the $0 \mathrm{nM}$ E2 group) in culture. E2 treatment did not affect the percentage of oligodendrocytes in culture (data not shown).

The results from the Western blotting analyses supported the immunofluorescence assay results. As shown in Figure 5, compared to NCs treated with $0,1,20$ and $50 \mathrm{nM}$ E2 did not affect the protein expression of Tuj1 or GFAP in culture. However, $10 \mathrm{nM}$ E2 treatment significantly increased the protein expression of Tuj1 (Figure $5 \mathrm{~A},{ }^{*} p=0.0111 \mathrm{vs}$. the $0 \mathrm{nM}$ E2 group) and decreased the protein expression of GFAP in culture (Figure $5 \mathrm{~B},{ }^{\star} p=0.0415 v s$. the $0 \mathrm{nM} \mathrm{E2}$ group). E2 treatment did not affect the protein expression of CNPase in culture (data not shown).

\section{Discussion}

It has been widely recognized that estrogens exhibit greater functionality (e.g., in the brain) than only the regulation of reproduction. Neurogenesis is an important brain function that exerts significant neuroprotective effects against several neurodegenerative diseases and is stimulated and regulated by estrogens (Bourque et al. 2009; Pike et al. 2009; Li et al. 2011). Tanapat et al. (1999) found that after injection with bromodeoxyuridine (BrdU), female mice had significantly more newly generated cells in the DG than did males. Additionally, within the estrous cycle, the number of newly

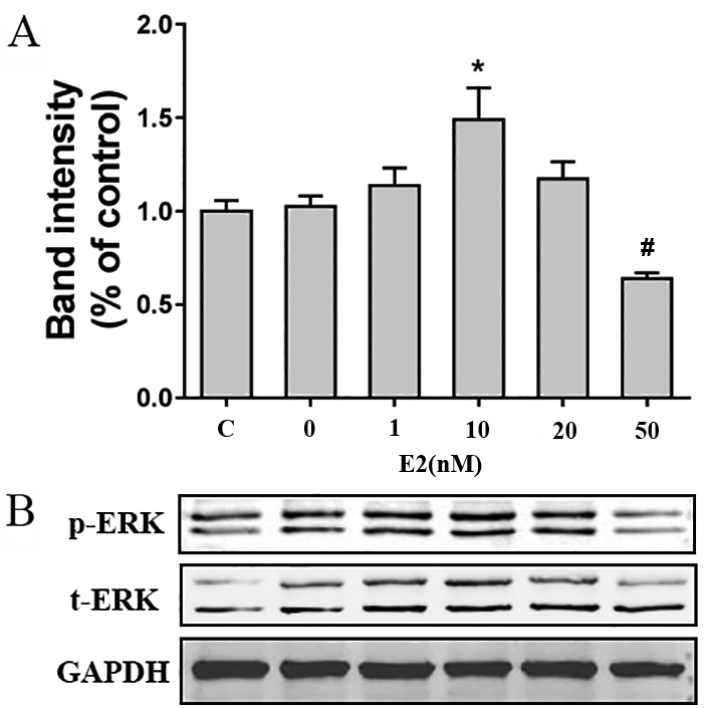

Figure 3. The effects of E2 treatment on p-ERK expression. A.The graph shows the results of the densitometric analysis of the p-ERK expression in different group. Data are shown as the mean \pm SD. One-way ANOVA: $\mathrm{F}=8.743, p=0.0011 ;{ }^{*} p=0.0174,{ }^{\#} p=0.048$ $v s$. the $0 \mathrm{nM}$ E2 group. B. Representative photographs showing the expression levels of $\mathrm{p}$-ERK and t-ERK proteins among various groups.

generated cells in the DG was 50\% higher in female mice during proestrus (high estradiol levels) than during estrus or diestrus (low estradiol levels). Furthermore, removal of the ovary diminished the number of newborn cells, and this effect was reversed by estrogen replacement (Tanapat et al. 1999). However, some researchers have reported opposite results. In another study, acute treatment with a moderate (not low or high) dose of estrogens rapidly increased newborn cell proliferation in ovariectomized animals, and chronic estrogen treatment for 3 weeks did not stimulate neurogenesis (Tanapat et al. 2005). In male rats, repeated estrogen administration did not significantly affect neurogenesis (Barker and Galea 2008). In another recent study, short-term treatment with estrogens decreased the rate of newly generated cells in the subventricular zone (SVZ) and olfactory bulb (OB) of adult female ovariectomized mice (Brock et al. 2010). These contrasting results indicate that the in vivo effects of estrogens on neurogenesis are complex. Several factors, such as the dose and timing of estrogen treatment, most likely influence the outcome.

Thus, many researchers have explored the effects and underlying mechanisms of action of estrogens on neurogenesis using NSCs. As estrogens exert their function by binding to ER $\alpha$ and ER $\beta$ and the newly identified GPR30 receptor, we first detected whether our cultured NSCs express these ERs. The immunofluorescence staining and Western blotting results revealed ER $\alpha, E R \beta$ and GPR30 to be highly 

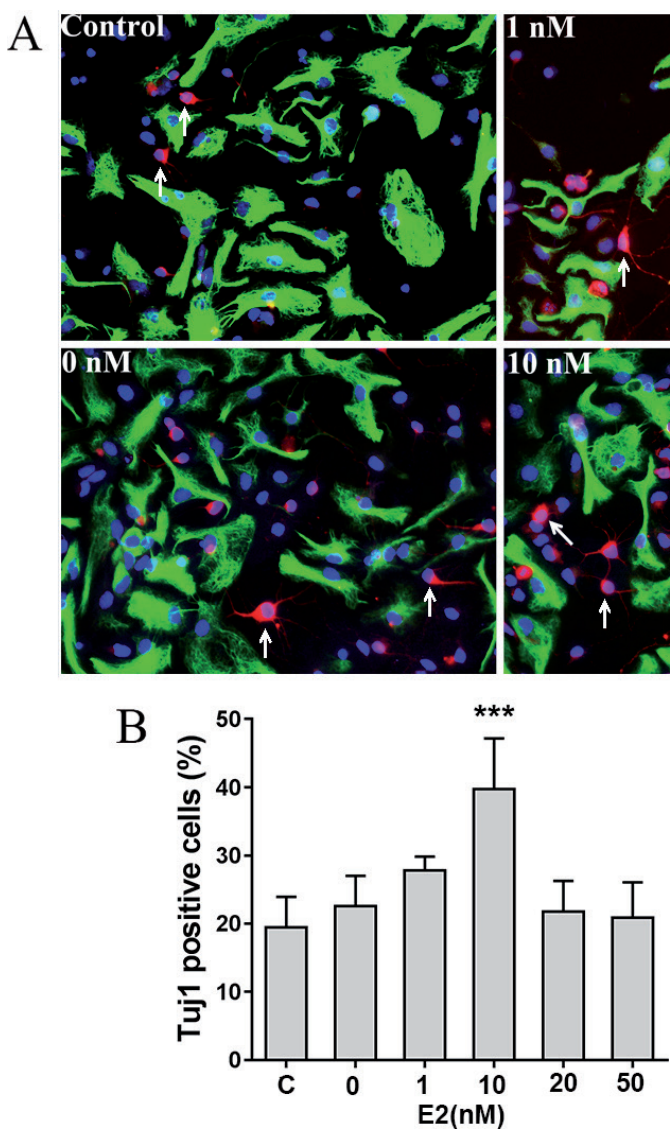
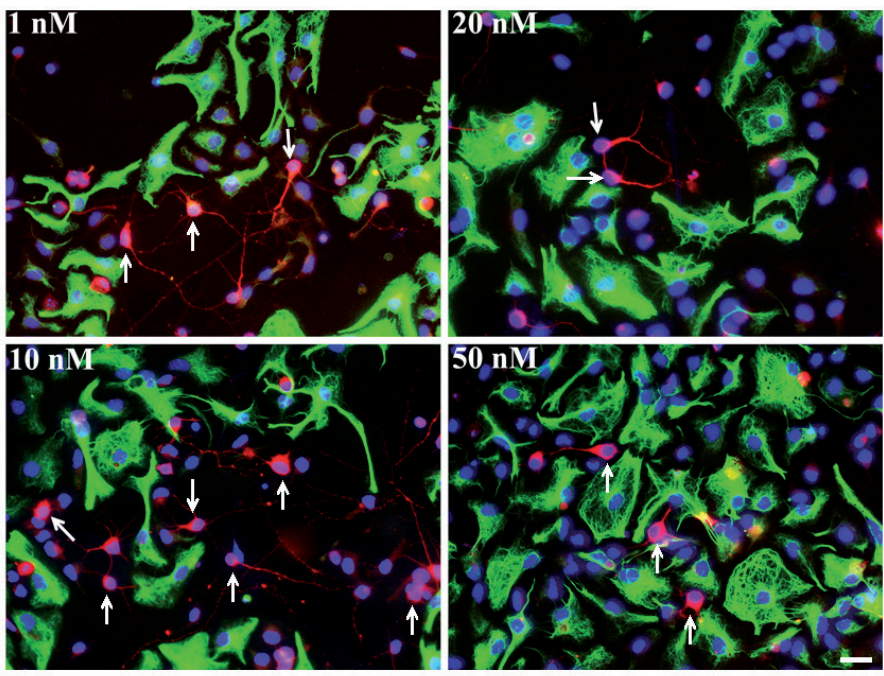

$\mathrm{C}$

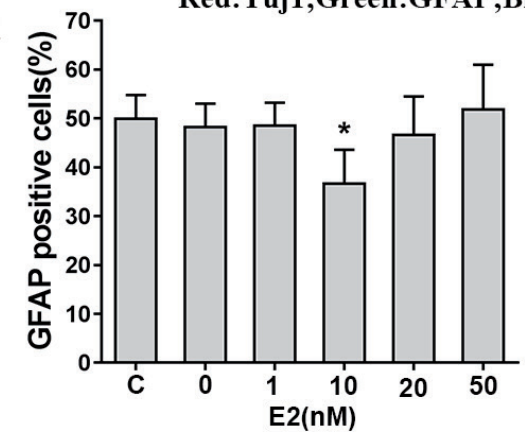

Figure 4. The effects of $\mathrm{E} 2$ treatment on the proliferation of NSCs, as assessed by immunofluorescence. A. Immunofluorescence images of NSCs stained for the neuronal marker Tuj1 (red) and the astrocytic marker GFAP (green) 7 days after E2 treatment. Bar: $20 \mu \mathrm{m}$. The white arrows represent Tuj1-stained neurons. B. The graph shows the results of the percentage of cells stained by the neuronal marker Tuj1 in different group. Data are expressed as the mean \pm SD. One-way ANOVA: $F=10.66,{ }^{* *} p=0.0001, v s$. the $0 \mathrm{nM} \mathrm{E2}$ group. $C$. The graph shows the results of the percentage of cells stained by the astrocytic marker GFAP in different group. Data are expressed as the mean \pm SD. One-way ANOVA: F=3.141, ${ }^{\star *} p=0.0484, v s$. the $0 \mathrm{nM}$ group. (See online version for color figure.)

expressed in cultured NSCs. A previous study demonstrated that NSCs expresses both ER $\alpha$ and ER $\beta$ (Brannvall et al. 2002). These results identify NSCs as a target for estrogens. Interestingly, we first found that the newly identified ER GPR30 was highly expressed in cultured NSCs. The GPR30 receptor is reported to be a novel ER uniquely localized in the endoplasmic reticulum (Funakoshi et al. 2006), and this receptor mediates rapid nongenomic effects of estrogen. This receptor is widely distributed and has numerous physiologic or pathologic functions in differentiated and mature brain cells (Chu et al. 2009; Grassi et al. 2009). However, the role of GPR30 in NSCs has not yet been explored and warrants further study. Furthermore, different neuronal cell types express different levels of ER. A recent study using quantitative PCR in primary cultured neurons and astrocytes found no difference between neurons and astrocytes in ER $\beta$ expression, though neurons did express higher levels of ERa (Piechota et al. 2017), which may be the basis of the various differentiation effects of estrogens on NSCs.

Next, we explored the role of different doses of E2 on the proliferation and differentiation of NSCs. The results from cell cycle analysis (flow cytometry) showed that 10 nM E2 treatment for 3 days significantly increased the proliferation of NSCs but that $50 \mathrm{nM}$ E2 markedly decreased the proliferation of NSCs. Brannvall et al. (2002) found that $10 \mathrm{nM}$ E2 significantly increased the number of BrdU-labeled cells among NSCs by 7\%, and this effect was inhibited by an ER antagonist. In another study, $10 \mathrm{nM} \mathrm{E2}$ increased the number of generated neurons (over 50\%) in mouse embryonic stem (ES) cells, with increased neurite branching (Murashov et al. 2004). To elucidate the underlying mechanism, we examined the effects of estrogens on phosphorylation-mediated activation of ERK1/2 (p-ERK1/2). The ERK signaling cascade 
A
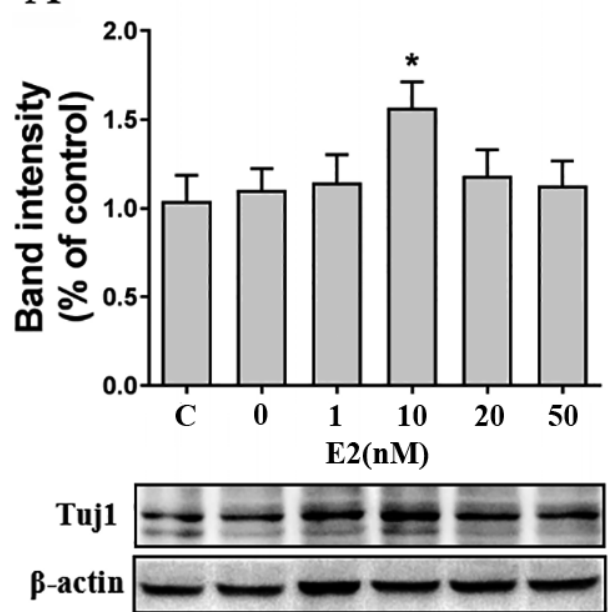

B

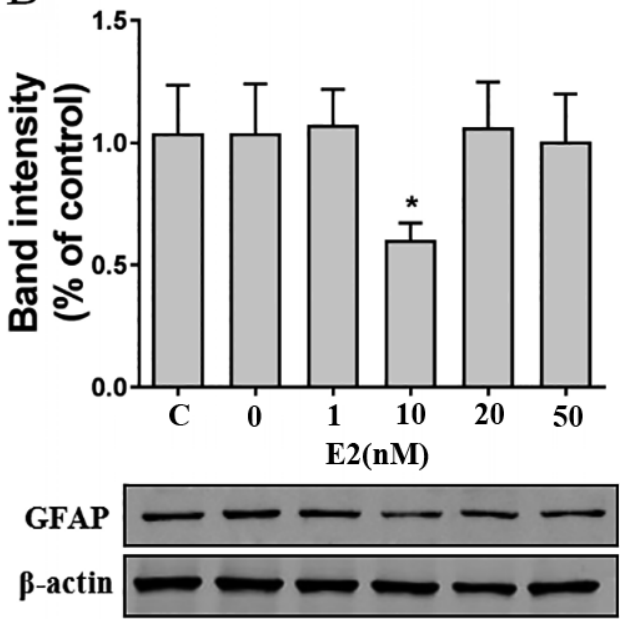

Figure 5. The effects of E2 treatment on the proliferation of NSCs, as assessed by Western blot analysis. A. Up: The graph shows the results of the densitometric analysis of the Tuj 1 expression in different groups. Data are shown as the mean \pm SD. One-way ANOVA: $\mathrm{F}=4.642,{ }^{\star} p=0.0111$ vs. the $0 \mathrm{nM}$ E2 group. Down: Representative images showing the expression of Tuj1 protein among various groups. B. Up: The graph shows the results of the densitometric analysis of the GFAP expression in different group. Data are shown as the mean \pm SD. One-way ANOVA: $F=3.13,{ }^{\star} p=0.0415$ vs. the $0 \mathrm{nM}$ E2 group. Down: Representative images showing the expression of the GFAP protein in groups.

regulates the proliferation (Xiao et al. 2007) of NSCs. In our study, 10 nM E2 treatment significantly increased p-ERK1/2 expression, whereas treatment with 50 nM E2 markedly decreased $\mathrm{p}$-ERK $1 / 2$ expression. Thus, the effects of estrogens on NSC proliferation may be mediated by the regulation of p-ERK1/2 expression.

NSCs from embryonic and adult brains can undergo differentiation into three major types of brain cells: neurons, astrocytes, and oligodendrocytes. The differentiation of NSCs into new neurons, which can be used in basic and translational studies for the treatment of neurodegenerative diseases, is particularly promising. However, control over their differentiation is still a critical obstacle. In this work, using immunofluorescence staining and Western blot analyses, we observed that $10 \mathrm{nM}$ E2 significantly stimulated the differentiation of NSCs into neurons and inhibited the differentiation of NSCs into astrocytes. The ratio of differentiated oligodendrocytes remained unchanged. In a previous study, 10 nM E2 increased the ratio of Tuj1-positive neurons to GFAP-positive glial cells in embryonic rat NSCs (determined by immunostaining), and this result demonstrated the influence of estrogens on neurogenesis during embryonic development (Brannvall et al. 2002). Icaritin, which is an ER modulator with neuroprotective effects (Wang et al. 2007), also facilitated the differentiation of mouse ES cells into the neuroectoderm and increased the proportion of Tuj1-positive cells (the number of GFAP-positive cells remained unchanged) (Wang et al. 2009). Altogether, these results suggest that estrogens affect the ratio of differentiated neurons from NSCs.

Currently, human NSC-replacement therapy has significant potential for treating neurodegenerative diseases. Human NSCs can give rise to neurons and glial cells in vitro and survive to differentiate into neurons in the rat brain (Fricker et al. 1999). E2 increased the number of dopaminergic (DA) neurons derived from human NSCs in vivo when these cells were grafted into mouse brains; this result also supports the role of estrogens during the transplantation of human NSCs for Parkinson's disease (Kishi et al. 2005). Additionally, recent studies have found that neuropeptides, including oxytocin, vasopressin, neuropeptide Y (NPY), and ghrelin, participate in the regulation of neurogenesis and differentiation (Bakos et al. 2016). Regardless, the role of these neuropeptides in estrogen-mediated neurogenesis remains unclear.

The electrophysiological properties of the medium spiny neurons (MSNs) have also been found to be sensitive to estradiol in a striatal region-specific manner: in both caudate-putamen and nucleus accumbens core, MSNs receive augmented excitatory synaptic input in females compared with that in males, and early-life exposure to estradiol is instrumental in the sexual differentiation of this property. These findings extend earlier mosaic models of brain sexuality, in which not only individual brain regions but also individual neuron types, in this instance MSNs, show differential degrees of feminization (Cao et al. 2018). 
In conclusion, our study demonstrates that NSCs are definitely a target for estrogens and that an appropriate dose of E2 (10 nM) can significantly increase the proliferation of NSCs and significantly stimulate NSCs to differentiate into neurons. These findings supplement our knowledge of the regulatory effects of estrogens on neurogenesis.

Conflict of interest. The authors declare no competing financial interests.

Acknowledgments. This study was supported by the National Natural Science Foundation of China (81801138 to Yulong Ma), the Beijing Municipal Science \& Technology Commission (no. 1811000017180022 to Hang Guo), the Beijing Natural Science Foundation (no. 7194321 to Lixia Zhang), the Miaopu Foundation of Chinese PLA General Hospital (no. 18KMM47 to Lixia Zhang), and the Young Scholar Research Grant of Chinese Anesthesiologist Association (no. 21700001 to Yulong Ma). We thank American Journal Experts (AJE) for assisting in the preparation of this manuscript.

\section{References}

Bakos J, Zatkova M, Bacova Z, Ostatnikova D (2016): The role of hypothalamic neuropeptides in neurogenesis and neuritogenesis. Neural Plast. 2016, 3276383 https://doi.org/10.1155/2016/3276383

Barker JM, Galea LA (2008): Repeated estradiol administration alters different aspects of neurogenesis and cell death in the hippocampus of female, but not male, rats. Neuroscience 152, 888-902 https://doi.org/10.1016/j.neuroscience.2007.10.071

Bourque M, Dluzen DE, di Paolo T (2009): Neuroprotective actions of sex steroids in Parkinson's disease. Front. Neuroendocrinol. 30, 142-157 https://doi.org/10.1016/j.yfrne.2009.04.014

Brannvall K, Korhonen L, Lindholm D (2002): Estrogen-receptordependent regulation of neural stem cell proliferation and differentiation. Mol. Cell. Neurosci. 21, 512-520 https://doi.org/10.1006/mcne.2002.1194

Brock O, Keller M, Veyrac A, Douhard Q, Bakker J (2010): Short term treatment with estradiol decreases the rate of newly generated cells in the subventricular zone and main olfactory bulb of adult female mice. Neuroscience 166, 368-376 https://doi.org/10.1016/j.neuroscience.2009.12.050

Cao J, Willett JA, Dorris DM, Meitzen J (2018): Sex differences in medium spiny neuron excitability and glutamatergic synaptic input: heterogeneity across striatal regions and evidence for estradiol-dependent sexual differentiation. Front. Endocrinol. (Lausanne) 9, 173 https://doi.org/10.3389/fendo.2018.00173

Chu Z, Andrade J, Shupnik MA, Moenter SM (2009): Differential regulation of gonadotropin-releasing hormone neuron activity and membrane properties by acutely applied estradiol: dependence on dose and estrogen receptor subtype. J. Neurosci. 29, 5616-5627

https://doi.org/10.1523/JNEUROSCI.0352-09.2009
Fricker RA, Carpenter MK, Winkler C, Greco C, Gates MA, Bjorklund A (1999): Site-specific migration and neuronal differentiation of human neural progenitor cells after transplantation in the adult rat brain. J. Neurosci. 19, 5990-6005 https://doi.org/10.1523/JNEUROSCI.19-14-05990.1999

Funakoshi T, Yanai A, Shinoda K, Kawano MM, Mizukami Y (2006): G protein-coupled receptor 30 is an estrogen receptor in the plasma membrane. Biochem. Biophys. Res. Commun. 346, 904-910 https://doi.org/10.1016/j.bbrc.2006.05.191

Grassi S, Frondaroli A, Dieni C, Scarduzio M (2009): Effects of 17beta-estradiol on synaptic plasticity in the rat medial vestibular nuclei. Acta Otolaryngol. 129, 390-394 https://doi.org/10.1080/00016480802566287

Jin K, Sun Y, Xie L, Peel A, Mao XO, Batteur S, Greenberg DA (2003): Directed migration of neuronal precursors into the ischemic cerebral cortex and striatum. Mol. Cell. Neurosci. 24, 171-189 https://doi.org/10.1016/S1044-7431(03)00159-3

Kishi Y, Takahashi J, Koyanagi M, Morizane A, Okamoto Y, Horiguchi S, Tashiro K, Honjo T, Fujii S, Hashimoto N (2005): Estrogen promotes differentiation and survival of dopaminergic neurons derived from human neural stem cells. J. Neurosci. Res. 79, 279-286 https://doi.org/10.1002/jnr.20362

Li J, Siegel M, Yuan M, Zeng Z, Finnucan L, Persky R, Hurn PD, McCullough LD (2011): Estrogen enhances neurogenesis and behavioral recovery after stroke. J. Cereb. Blood Flow Metab. 31, 413-425 https://doi.org/10.1038/jcbfm.2010.181

Majewski L, Sobczak M, Wasik A, Skowronek K, Redowicz MJ (2011): Myosin VI in PC12 cells plays important roles in cell migration and proliferation but not in catecholamine secretion. J. Muscle Res. Cell Motil. 32, 291-302 https://doi.org/10.1007/s10974-011-9279-0

Murashov AK, Pak ES, Hendricks WA, Tatko LM (2004): 17betaEstradiol enhances neuronal differentiation of mouse embryonic stem cells. FEBS Lett. 569, 165-168 https://doi.org/10.1016/j.febslet.2004.05.042

Nie H, Peng Z, Lao N, Dong H, Xiong L (2013): Effects of sevoflurane on self-renewal capacity and differentiation of cultured neural stem cells. Neurochem. Res. 38, 1758-1767 https://doi.org/10.1007/s11064-013-1074-4

Piechota M, Korostynski M, Golda S, Ficek J, Jantas D, Barbara Z, Przewlocki R (2017): Transcriptional signatures of steroid hormones in the striatal neurons and astrocytes. BMC Neurosci. 18, 37 https://doi.org/10.1186/s12868-017-0352-5

Pike CJ, Carroll JC, Rosario ER, Barron AM (2009): Protective actions of sex steroid hormones in Alzheimer's disease. Front. Neuroendocrinol. 30, 239-258 https://doi.org/10.1016/j.yfrne.2009.04.015

Rettberg JR, Yao J, Brinton RD (2014): Estrogen: a master regulator of bioenergetic systems in the brain and body. Front. Neuroendocrinol. 35, 8-30 https://doi.org/10.1016/j.yfrne.2013.08.001

Shao B, Cheng Y, Jin K (2012): Estrogen, neuroprotection and neurogenesis after ischemic stroke. Curr. Drug Targets 13, 188-198 
https://doi.org/10.2174/138945012799201702

Tanapat P, Hastings NB, Gould E (2005): Ovarian steroids influence cell proliferation in the dentate gyrus of the adult female rat in a dose- and time-dependent manner. J. Comp. Neurol. 481, 252-265

https://doi.org/10.1002/cne.20385

Tanapat P, Hastings NB, Reeves AJ, Gould E (1999): Estrogen stimulates a transient increase in the number of new neurons in the dentate gyrus of the adult female rat. J. Neurosci. 19, 5792-5801 https://doi.org/10.1523/JNEUROSCI.19-14-05792.1999

Wang Z, Wang H, Wu J, Zhu D, Zhang X, Ou L, Yu Y, Lou Y (2009): Enhanced co-expression of beta-tubulin III and choline acetyltransferase in neurons from mouse embryonic stem cells promoted by icaritin in an estrogen receptor-independent manner. Chem. Biol. Interact. 179, 375-385

https://doi.org/10.1016/j.cbi.2008.12.007
Wang Z, Zhang X, Wang H, Qi L, Lou Y (2007): Neuroprotective effects of icaritin against beta amyloid-induced neurotoxicity in primary cultured rat neuronal cells via estrogen-dependent pathway. Neuroscience 145, 911-922

https://doi.org/10.1016/j.neuroscience.2006.12.059

Workman JL, Barha CK, Galea LA (2012): Endocrine substrates of cognitive and affective changes during pregnancy and postpartum. Behav. Neurosci. 126, 54-72 https://doi.org/10.1037/a0025538

Xiao Z, Kong Y, Yang S, Li M, Wen J, Li L (2007): Upregulation of Flk1 by bFGF via the ERK pathway is essential for VEGF-mediated promotion of neural stem cell proliferation. Cell Res. 17, 73-79 https://doi.org/10.1038/sj.cr.7310126

Received: December 12, 2018

Final version accepted: May 5, 2019 